\title{
SEGMENT-PRESERVING MAPS OF PARTIAL ORDERS
}

\author{
BY \\ MARTIN AIGNER( $\left.{ }^{(}\right)$AND GEERT PRINS
}

\begin{abstract}
A bijective map from a partial order $P$ to a partial order $Q$ is defined to be segment-preserving if the image of every segment in $P$ is a segment in $Q$. It is proved that a partial order $P$ with 0 -element admits nontrivial segment-preserving maps if and only if $\boldsymbol{P}$ is decomposable in a certain sense. By introducing the concept of "strong" segment-preserving maps further insight into the relations between segment-preserving maps and decompositions of partial orders is obtained.
\end{abstract}

I. Introduction. Let $P$ be a partial order, then for $a, b \in P$ with $a \leqq b$ we define the segment $[a, b]$ as the set of all $x \in P$ with $a \leqq x \leqq b$. Let $P, Q$ be partial orders and suppose the function $\phi$ maps the elements one-one onto those of $Q$. The map $\phi$ is called segment-preserving if for any segment $[a, b] \subseteq P$ the set $\{\phi(x): x \in[a, b]\}$ forms a segment of $Q$. The map $\phi$ is an isomorphism if either for all $a, b \in P, a<b$ if and only if $\phi(a)<\phi(b)$, or for all $a, b \in P, a<b$ if and only if $\phi(b)<\phi(a)$. Clearly an isomorphism is segment-preserving and we shall henceforth refer to isomorphisms as trivial maps.

The purpose of this note is an investigation of all possible segment-preserving maps of any partial order $P$ which possesses a zero-element $\left({ }^{2}\right)$. Closely related to our work is a conjecture of G.-C. Rota, later proved by A. M. Gleason (unpublished) that a partially ordered set is essentially determined by the order of inclusion on its segments. Let $P$ be a partially ordered set with zero-element and $Q$ an arbitrary partial order such that $P$ and $Q$ have isomorphic orders on their segments. This isomorphism, restricted to the trivial segments, i.e. the elements of $P$, is clearly a segment-preserving map $\phi$ from $P$ onto $Q$, and $\phi^{-1}$ is also segmentpreserving. It now follows immediately from our Theorem 5 that if $\phi$ is nontrivial, then there exist $U, V$ such that $P=U \otimes V, Q=U^{*} \otimes V$, where $U^{*}$ is the partial order dual to $U$ and $\otimes$ stands for the direct product. This result is obtained independently by Gleason, who has also extended it to a more intricate result on arbitrary partial orders.

Received by the editors October 27, 1970.

AMS 1970 subject classifications. Primary 06A10.

Key words and phrases. Segment-preserving map (bijection) of partial order, semidecomposition and semiproducts of partial orders.

( $\left.{ }^{1}\right)$ The research in this article was partially sponsored by the U.S. Air Force Office of Scientific Research under contract No. AFOSR-68-1406.

${ }^{2}$ ) Alternately we may assume the existence of a unit-element, developing the ensuing theory in an analogous fashion. 
After preliminary remarks we introduce semiproducts and semidecompositions of partial orders in $\S I I I$ and proceed to relate them to the structure of partial orders which admit nontrivial maps (§IV). The final section is devoted to a discussion of strongly segment-preserving maps and the determination of those partial orders for which every segment-preserving map is of that type.

Throughout the paper the term map shall always mean a segment-preserving map. A map will mostly be denoted by $\phi$ and we shall frequently use the abbreviation $\phi(x)=x^{\prime}$ for $x \in P . \phi[a, b]=\left[u^{\prime}, v^{\prime}\right]$ means the segment $[a, b]$ is mapped by $\phi$ onto the segment $\left[u^{\prime}, v^{\prime}\right]$. When we refer to the set of elements of a partial order $P$, regardless of the ordering, we shall use the symbol $E(P)$. If, for two partial orders $P, Q, E(P) \subseteq E(Q)$ and $a \leqq b$ in $P$ iff $a \leqq b$ in $Q$ hold, we write $P \subseteq Q$. The notation $x \diamond y$ indicates $x, y$ are incomparable elements.

II. Preliminary results. Throughout this section $P$ will denote a partial order, $\phi$ a map acting on $P$.

Proposition 1. Let $P$ have a zero-element 0 . If $\phi[0, b]=\left[0^{\prime}, b^{\prime}\right]$ for all $b \in P$ (or, similarly, if $\phi[0, b]=\left[b^{\prime}, 0^{\prime}\right]$ for all $\left.b \in P\right)$ then $\phi$ is trivial.

Proof. We first verify $x \diamond y$ iff $x^{\prime} \diamond y^{\prime}$ for $x, y \in P$. If, say, $x<y$ then $x \in[0, y]$ and hence $x^{\prime} \in\left[0^{\prime}, y^{\prime}\right]$ (or $x^{\prime} \in\left[y^{\prime}, 0^{\prime}\right]$ ). In either case $x^{\prime}, y^{\prime}$ are comparable in $\phi(P)$. Conversely $x^{\prime}<y^{\prime}$ implies $x^{\prime} \in\left[0^{\prime}, y^{\prime}\right]$ (or $y^{\prime} \in\left[x^{\prime}, 0^{\prime}\right]$ ) and hence $x \in[0, y]$ (or $y \in[0, x])$. Thus $x, y$ are comparable. Now the hypothesis plainly implies that $\phi$ either preserves all relations in $P$ or inverts all relations, and hence is an isomorphism.

Proposition 2. Let $P=[a, b]$ be a segment. Suppose $\phi[a, b]=\left[u^{\prime}, v^{\prime}\right] \neq\left[a^{\prime}, b^{\prime}\right]$, $\neq\left[b^{\prime}, a^{\prime}\right]$, then

(i) $u \diamond v$ in $P$,

(ii) $a^{\prime} \diamond b^{\prime}$ in $\phi(P)$,

(iii) $u, v$ are both different from $a, b$.

Proof. Assume $u, v$ are comparable. Then $\phi[u, v]$ (or $\phi[v, u])=\left[u^{\prime}, v^{\prime}\right]=\phi[a, b]$, and hence $[u, v]$ (or $[v, u])=[a, b]$, a contradiction. Clearly (i) now implies (iii). If $a^{\prime}, b^{\prime}$ are comparable, then $a^{\prime} \in \phi[u, b]$ or $b^{\prime} \in \phi[a, u]$ which implies $a \in[u, b]$ or $b \in[a, u]$, respectively. But this means $u=a$ or $u=b$, contradicting (iii).

Proposition 3. Let $P=[a, b]$ be a segment and suppose $\phi$ maps $P$ nontrivially. Then $\phi[a, b] \neq\left[a^{\prime}, b^{\prime}\right], \neq\left[b^{\prime}, a^{\prime}\right]$, and hence (1) holds.

Proof. Let us assume $\phi[a, b]=\left[a^{\prime}, b^{\prime}\right]$ (the argument for the other case being entirely analogous). By Proposition 1 , there exists $c \in P$ with $\phi[a, c] \neq\left[a^{\prime}, c^{\prime}\right]$, i.e., $\phi[a, c]=\left[a^{\prime}, d^{\prime}\right]$ with $c \neq d$. The segment $[a, c]$ now satisfies the hypothesis of the preceding proposition, yet (1) (iii) is violated. 
Corollary 1. Let $P$ have a zero-element. For any segment $[a, b] \subseteq P$ and any $\phi$ we have

either $\phi[a, b]=\left[a^{\prime}, b^{\prime}\right]$ or $=\left[b^{\prime}, a^{\prime}\right]$, in which case $\phi$ maps $[a, b]$ trivially,

(2) or $\phi[a, b]=\left[u^{\prime}, v^{\prime}\right]$ with $u, v$ both different from $a, b$, in which case $\phi$ maps $[a, b]$ nontrivially.

Proposition 4. Let $P=[a, b]$ be a segment and $\phi$ a nontrivial map with $\phi[a, b]$ $=\left[u^{\prime}, v^{\prime}\right]$. Then for $z \in P$,

$$
\begin{gathered}
z \geqq u, z \geqq v \Rightarrow z=b, \\
z \leqq u, z \leqq v \Rightarrow z=a, \\
z^{\prime} \geqq a^{\prime}, z^{\prime} \geqq b^{\prime} \Rightarrow z^{\prime}=v^{\prime}, \\
z^{\prime} \leqq a^{\prime}, z^{\prime} \leqq b^{\prime} \Rightarrow z^{\prime}=u^{\prime} .
\end{gathered}
$$

Proof. Assume $z \geqq u, z \geqq v$. Then $z^{\prime} \in \phi[u, b]=\left[u^{\prime}, b^{\prime}\right]$ by (2), and similarly $z^{\prime} \in\left[b^{\prime}, v^{\prime}\right]$. But this implies $z^{\prime}=b^{\prime}$, and thus $z=b$. The remaining statements are proved in a similar fashion.

Proposition 5. Let $P=[a, b]$ be a segment and $\phi$ a nontrivial map with $\phi[a, b]$ $=\left[u^{\prime}, v^{\prime}\right]$. Then for $z \in P$,

$$
z \diamond u \text { and } z \diamond v \text { iff } z^{\prime} \diamond a^{\prime} \text { and } z^{\prime} \diamond b^{\prime} .
$$

Proof. The assumption $z \diamond u, z \diamond v$ implies $z \notin[a, u],[u, b],[a, v],[v, b]$. Hence by (2) we infer $z^{\prime} \notin\left[u^{\prime}, a^{\prime}\right],\left[u^{\prime}, b^{\prime}\right],\left[a^{\prime}, v^{\prime}\right],\left[b^{\prime}, v^{\prime}\right]$, or equivalently $z^{\prime} \diamond a^{\prime}, z^{\prime} \diamond b^{\prime}$. The converse is now obvious.

III. Semiproducts of partial orders. We begin by recalling the following concepts (see e.g. [1]). Let $P$ be a partial order. A lower semi-ideal $I$ of $P$ is a suborder of $P$, i.e., $E(I) \subseteq E(P)$, endowed with the same partial order, such that if $x \in I$ and $y \leqq x$ then $y \in I$. Let $\left\{P_{\alpha}: \alpha \in A\right\}$ be a family of pairwise disjoint partial orders with zero-elements $0_{\alpha}(\alpha \in A)$. The direct sum $\sum_{\alpha \in A} P_{\alpha}$ is the partial order with $E\left(\sum_{\alpha \in A} P_{\alpha}\right)$ $=\bigcup_{\alpha \in A} E\left(P_{\alpha}\right)$ such that $p \leqq q$ in $\sum_{\alpha \in A} P_{\alpha}$ iff for some $\alpha \in A, p, q \in P_{\alpha}$ and $p \leqq q$ in $P_{\alpha}$. The direct product $\prod_{\alpha \in A} P_{\alpha}$ is the partial order defined on the set of all $A$-tuples with coordinates from $P_{\alpha}(\alpha \in A)$, i.e., $E\left(\prod_{\alpha \in A} P_{\alpha}\right)=\left\{p=\left(\cdots p_{\alpha} \cdots\right): p_{\alpha} \in P_{\alpha}, \alpha \in A\right\}$, such that $p \leqq q$ in $\prod_{\alpha \in A} P_{\alpha}$ iff $p_{\alpha} \leqq q_{\alpha}$ for all $\alpha \in A$. For all $\alpha \in A$ and all $p_{\alpha} \in P_{\alpha}$ we henceforth identify the element $p_{\alpha}$ with the $A$-tuple $\left(\cdots p_{\alpha} \cdots\right), p_{\beta}=0_{\beta}$ for $\beta \neq \alpha$. With this agreement $\sum_{\alpha \in A} P_{\alpha} \subseteq \prod_{\alpha \in A} P_{\alpha}$.

Definition. Let $\left\{P_{\alpha}: \alpha \in A\right\}$ be a family of pairwise disjoint partial orders with zero-elements $0_{\alpha}(\alpha \in A)$. A semiproduct of the $P_{\alpha}$ 's is a lower semi-ideal $I$ of the direct product such that $\sum_{\alpha \in A} P_{\alpha} \subseteq I \subseteq \prod_{\alpha \in A} P_{\alpha}$.

Both the direct sum and the direct product clearly are examples of semiproducts. To facilitate the reference we shall denote semiproducts, the direct sum and the direct product of a family $\left\{P_{\alpha}: \alpha \in A\right\}$ by $\prod_{\alpha \in A} P_{\alpha}, \prod_{\alpha \in A}^{\oplus} P_{\alpha}, \prod_{\alpha \in A}^{\otimes} P_{\alpha}$, respectively. We call the $P_{\alpha}$ 's the factors of the product. When we write down the factors of a 
product we shall use the notation (e.g., in the case of two factors $U, V) U \times V$, $U \oplus V, U \otimes V$, respectively.

In much the same way as for direct products, we now introduce semidecompositions of partial orders. In defining semiproducts the factors were assumed to be pairwise disjoint. We now identify all the zero-elements. This clearly can be done without causing any ambiguity. Let $P$ be a partial order with zero-element 0 and $\left\{P_{\alpha} \subseteq P: \alpha \in A\right\}$ a family of suborders containing 0 , which are otherwise pairwise disjoint. With $P$ and $\left\{P_{\alpha}\right\}$ we may associate a unique semiproduct $\prod_{\alpha \in A}^{P} P_{\alpha}$ by the rule $\left(\cdots p_{\alpha} \cdots\right) \in E\left(\prod_{\alpha \in A}^{P} P_{\alpha}\right)$ iff there exists $p \in P$ such that $p_{\alpha} \leqq p$ for all $\alpha \in A$.

Definition. The family $\left\{P_{\alpha} \subseteq P: \alpha \in A\right\}$ is called a semidecomposition of $P$ if there exists an order-preserving isomorphism $\sigma$ from $P$ onto $\prod_{\alpha \in A}^{P} P_{\alpha}$ such that $\sigma\left(p_{\alpha}\right)=p_{\alpha}$ for all $\alpha \in A$ and all $p_{\alpha} \in P_{\alpha}$. We then write $P=\prod_{\alpha \in A} P_{\alpha}$ and identify $p \equiv \sigma(p)$ for all $p \in P$.

From the definition of a semiproduct it readily follows that every factor $P_{\alpha}$ is a lower semi-ideal of $\prod_{\alpha \in A} P_{\alpha}$. Again from the definition of $\prod_{\alpha \in A} P_{\alpha}$ it is clear that $\left(\cdots p_{\alpha} \cdots\right)$ is the unique join of the elements $p_{\alpha} \in P_{\alpha}$. Hence if $p=\left(\cdots p_{\alpha} \cdots\right)$, then $p_{\alpha}$ is the maximal element of the partial order $\left\{x \in P_{\alpha}: x \leqq p\right\}$. As a consequence we note that if for a set $\left\{x_{i} \in P_{\alpha}: i \in \mathscr{I}\right\}$ there exists $p \in P$ with $x_{i} \leqq p$ for all $i \in \mathscr{I}$, then there exists $p^{\prime} \in P_{\alpha}$ with the same property.

With these preparations the following statement is immediate.

Lemma. If $P=\prod_{\alpha \in A} P_{\alpha}$ and $P_{\alpha}=\prod_{\beta \in B_{\alpha}} P_{\alpha, \beta}$ for $\alpha \in A$, then $P=\prod_{\alpha} \prod_{\beta} P_{\alpha, \beta}$.

Proposition 6. Let $P$ have a zero-element 0. Suppose $P=\prod_{\alpha \in A} P_{\alpha}$ and $P=\prod_{\beta \in B} Q_{\beta}$ are two semidecompositions of $P$. Then $P_{\alpha}=\prod_{\beta \in B}\left(P_{\alpha} \cap Q_{\beta}\right)$ for $\alpha \in A$, and hence by the Lemma $P=\prod_{\alpha, \beta}\left(P_{\alpha} \cap Q_{\beta}\right)$.

Proof. Let $p \in P_{\alpha}$ and suppose $p=\left(\cdots p_{\beta} \cdots\right)$ in $\prod_{\beta \in B} Q_{\beta}$. Since $P_{\alpha}$ is a lower semi-ideal, $p_{\beta} \in P_{\alpha}$ for all $\beta \in B$ and hence $P_{\alpha} \subseteq \prod_{\beta \in B}^{P_{\alpha}}\left(P_{\alpha} \cap Q_{\beta}\right)$. On the other hand, let $p \in \prod_{\beta \in B}^{P_{\alpha}}\left(P_{\alpha} \cap Q_{\beta}\right)$, then $p=\left(\cdots q_{\beta} \cdots\right)$ with $q_{\beta} \in P_{\alpha} \cap Q_{\beta}$. If $p=\left(\cdots p_{\alpha} \cdots\right)$ in $\prod_{\alpha \in A} P_{\alpha}$, then $q_{\beta} \leqq p_{\alpha}$ for all $\beta \in B$, since $p_{\alpha}$ is the maximal element of $P_{\alpha}$ below $p$. But $p$ is the unique join of the $q_{\beta}$ 's, thus $p=p_{\alpha}$, and the conclusion follows.

Every partial order $P$ admits the trivial semidecomposition $P=P \times\{0\}$. If there are no others, $P$ is called semi-indecomposable.

Corollary 2. If there exists a semidecomposition $\left({ }^{3}\right) P=\prod_{\alpha \in A} P_{\alpha}$ of $P$ into semiindecomposable factors $P_{\alpha} \neq\{0\}$, then this semidecomposition is unique (up to order).

Proposition 7. Let $P=\prod_{\alpha \in A} P_{\alpha}$ be a semidecomposition of $P$. If $A=\bigcup_{i \in \mathscr{I}} A_{i}$ is any partitioning of the index-set, then $P=\prod_{i \in \mathscr{I}}\left(\prod_{\alpha \in A_{t}} P_{\alpha}\right)$.

(3) The question of existence of a decomposition is unresolved in general, even in the case of direct products. 
Proof. Let $p \in P$ and $p=\left(\cdots p_{\alpha} \cdots\right)$ in $\prod_{\alpha \in A} P_{\alpha}$. The function $\sigma(p)=\left(\cdots p_{i} \cdots\right)$, where $p_{i}=\left(\cdots p_{\alpha} \cdots\right)$ with $p_{\beta}=0$ for $\beta \notin A_{i}$, maps $P$ onto $\prod_{i \in \mathscr{I}}^{P}\left(\prod_{\alpha \in A_{i}} P_{\alpha}\right)$ and satisfies all the requirements.

Corollary 3. If there exists a semidecomposition $P=\prod_{\alpha \in A} P_{\alpha}$ of $P$ into semiindecomposable factors, then any semidecomposition of $P$ into two factors $P=U \times V$ is given by $U=\prod_{\beta \in B} P_{\beta}, V=\prod_{\gamma \in \Gamma} P_{\gamma}$ for some partition $A=B \cup \Gamma$ of the index-set $A$.

For brevity, we shall shorten the terms semiproduct, semidecomposition, etc., to $S$-product, $S$-decomposition, etc., in the remainder of the paper.

\section{The main theorem.}

THEOREM 1. Let $P$ be a partial order with zero-element 0 and suppose $\phi$ maps $P$ nontrivially. Then

$$
P=U \times V,
$$

where $U=\left\{x \in P: x^{\prime} \leqq 0^{\prime}\right\}, V=\left\{x \in P: x^{\prime} \geqq 0^{\prime}\right\}$.

Proof. First we note $U \cap V=\{0\}$. By Proposition 1 and (2) it is clear that $U, V$ are mapped trivially with $\phi(U) \cong U^{*}, \phi(V) \cong V$, and that a segment $[0, z] \subseteq P$ is mapped trivially iff $z \in U$ or $z \in V$. Let $p \in P$ be arbitrary and suppose

$$
\phi[0, p]=\left[p_{1}^{\prime}, p_{2}^{\prime}\right],
$$

where $p_{1} \in U, p_{2} \in V$. We define the function $\sigma$ on $P$ by

$$
\sigma(p)=\left(p_{1}, p_{2}\right)
$$

and proceed to show that $\sigma$ is the required isomorphism from $P$ onto $U \mathbf{X}^{P} V$.

If $p \in U$ or $p \in V$, then $[0, p]$ is mapped trivially, hence by (6) and (7) $\sigma(p)=p$. Any pair $\left(p_{1}, p_{2}\right)$ with $p_{1} \in U, p_{2} \in V$, which is the image under (7) of some $p \in P$, belongs to $U \mathbf{X}^{P} V$, since by (6) $p_{1} \leqq p, p_{2} \leqq p$. Furthermore, it is clear that $\sigma$ maps distinct elements $p, q$ onto distinct pairs.

To prove the range of $\sigma$ is all of $U \mathbf{X}^{P} V$ let $r \in U, s \in V$ be such that there exists $p \in P$ with $p \geqq r, p \geqq s$. By our previous remarks, we may assume $r>0, s>0$. We have $r, s \in[0, p]$, and hence $r^{\prime}, s^{\prime} \in\left[p_{1}^{\prime}, p_{2}^{\prime}\right]$, where $\sigma(p)=\left(p_{1}, p_{2}\right)$. If $r=p_{1}, s=p_{2}$, we are finished. Suppose, w.l.o.g., $r \neq p_{1}$, then $p_{1}^{\prime}<r^{\prime}$, and thus $r<p_{1}$. Consider the segment $[r, p]$. Since $p_{1} \in[r, p]$ and $p_{1}^{\prime}<r^{\prime}, p_{1}^{\prime}<p^{\prime}$, by (2) $\phi$ maps $[r, p]$ nontrivially, say

$$
\phi[r, p]=\left[p_{1}^{\prime}, q^{\prime}\right] .
$$

By (1), $p_{1} \diamond q$ and thus if $p_{2} \diamond q$, we would conclude, by (4), $p^{\prime} \diamond q^{\prime}$, a contradiction to (8). Hence $p_{2}, q$ are comparable. If $q \leqq p_{2}$ then, by (8), $r<p_{2}$, and since also $r<p_{1}$, we deduce, by (3), $r=0$, a contradiction. Therefore $p_{2}<q$, and thus $r, p_{2} \in[0, q]$. Since $p_{1} \diamond q$ and $p_{1}^{\prime}<q^{\prime}, q \notin U$. Similarly $p_{2}<q$ and $q^{\prime}<p_{2}^{\prime}$ imply $q \notin V$. Hence $\phi$ maps the segment $[0, q]$ nontrivially. Applying (3) to $r^{\prime}<0^{\prime}, r^{\prime}<q^{\prime}$ (by (8)) 
and to $0^{\prime}<p_{2}^{\prime}, q^{\prime}<p_{2}^{\prime}$, we infer $\phi[0, q]=\left[r^{\prime}, p_{2}^{\prime}\right]$, and thus $\sigma(q)=\left(r, p_{2}\right)$. If $s=p_{2}$, we are finished. Otherwise $s<p_{2}$, and we repeat the argument with $s, q, p_{2}$ in place of $r, p, p_{1}$. This way we finally arrive at an element $t \in P$ with $\sigma(t)=(r, s)$.

To prove $\sigma$ is order-preserving take $p, q \in P$ with $\sigma(p)=\left(p_{1}, p_{2}\right), \sigma(q)=\left(q_{1}, q_{2}\right)$ and $p \leqq q$. Then $[0, p] \subseteq[0, q]$, and hence $\left[p_{1}^{\prime}, p_{2}^{\prime}\right] \subseteq\left[q_{1}^{\prime}, q_{2}^{\prime}\right]$. But this means $q_{1}^{\prime} \leqq p_{1}^{\prime}$, $p_{2}^{\prime} \leqq q_{2}^{\prime}$, or equivalently $p_{1} \leqq q_{1}, p_{2} \leqq q_{2}$. The whole argument is clearly reversible, and the theorem follows.

V. Strongly segment-preserving maps. According to Theorem 1, with every map $\phi$ on $P$ we may associate by (5) a uniquely determined ordered $S$-decomposition $P=U \times V$ of $P$. We express this correspondence in symbols $[\phi]=[U, V]$. Conversely, given an arbitrary $S$-decomposition $P=U \times V$, we ask whether there exists a map $\phi$ with $[\phi]=[U, V]$.

Definition. A map $\phi$ on the partial order $P$ is called strongly segment-preserving (a strong map, for short) if the pre-image of every segment $Q$ of $\varphi(P)$ has a zeroelement $0_{Q}$ in $P$.

THEOREM 2. Let $P$ be a partial order with zero-element 0 . For any $S$-decomposition $P=U \times V$ of $P$, there exists a unique strong map $\phi(U, V)$ such that $[\phi(U, V)]$ $=[U, V] . \phi(U, V)$ is given by

$$
\phi(U, V)(x, y) \leqq \phi(U, V)(r, s) \quad \text { iff } \quad r \leqq x, y \leqq s
$$

where $r, x \in U, s, y \in V$.

Proof. Let $P=U \times V$ be an arbitrary but fixed $S$-decomposition of $P$ and $\phi(U, V)=\psi$ as given by (9). The definition of $\psi$ clearly implies that $\psi(P)$ is a partial order and $[\psi]=[U, V]$. To prove $\psi$ is segment-preserving let $[(r, y),(x, s)] \subseteq P$ be a segment. Then

$$
\psi[(r, y),(x, s)]=\{\psi(k, l): r \leqq k \leqq x, y \leqq l \leqq s\}=[\psi(x, y), \psi(r, s)] \quad \text { by }(9)
$$

and the fact that $(r, y),(x, s) \in P, r \leqq x, y \leqq s$ and hence $(x, y),(r, s) \in P$. This proves $\psi$ is a map.

If $Q=[\psi(x, y), \psi(r, s)]$ is any segment of $\psi(P)$, then $(r, y) \in P$ (by (9)) and is minimal in the pre-image of $Q$. Hence $(r, y)=0_{Q}$, and $\psi$ is a strong map.

Let $\phi$ now be an arbitrary strong map with $[\phi]=[U, V]$. We have to verify (9). If $(x, y),(r, s) \in P$ with $r \leqq x, y \leqq s$, then $(r, y) \in P$ and

$$
(r, y) \in[r,(r, s)], \quad(r, y) \in[y,(x, y)] .
$$

Since, by (6), $r^{\prime} \leqq(r, s)^{\prime},(x, y)^{\prime} \leqq y^{\prime}$, we deduce from (1) and (2) that the segments in (10) are mapped trivially, and thus $(x, y)^{\prime} \leqq(r, y)^{\prime} \leqq(r, s)^{\prime}$. Conversely, let $(x, y)^{\prime}$ $\leqq(r, s)^{\prime}$ and consider the segment $Q=\left[(x, y)^{\prime},(r, s)^{\prime}\right] \subseteq \phi(P)$ with $0_{Q}=(a, b)$. By (6),

$$
\begin{aligned}
& x^{\prime} \leqq(x, y)^{\prime} \leqq(a, b)^{\prime} \leqq b^{\prime}, \\
& a^{\prime} \leqq(a, b)^{\prime} \leqq(r, s)^{\prime} \leqq s^{\prime} .
\end{aligned}
$$


Since by assumption $(a, b) \leqq(x, y),(a, b) \leqq(r, s)$, we infer $(x, b),(a, s) \in P$ and by (6)

$$
\phi[0,(x, b)]=\left[x^{\prime}, b^{\prime}\right], \quad \phi[0,(a, s)]=\left[a^{\prime}, s^{\prime}\right] .
$$

Together with (11) this implies $(x, y) \leqq(x, b),(r, s) \leqq(a, s)$. By the minimality of $(a, b)$ we now deduce $y=b, r=a$ and thus $r \leqq x, y \leqq s$. This means $\phi=\phi(U, V)$, and the proof is complete.

In the preceding proof it was shown that the right-hand-to-left-hand implication, spelled out in (9), holds for any map. Furthermore it follows from the argument that we may also relax the reverse implication to obtain the following characterization of strong maps:

A map $\phi$ with $[\phi]=[U, V]$ is strong iff

whenever $\phi(x, y) \leqq \phi(r, s)$ then there exists $(a, b)$ with $(a, b) \leqq(x, y)$,

$(a, b) \leqq(r, s)$ such that $\phi(x, y) \leqq \phi(a, b) \leqq \phi(r, s)$.

In the remainder of the paper we present characterizations of partial orders which only admit strong maps. $P$ will always denote a partial order, $\phi$ a map.

THEOREM 3. Let $P$ have a zero-element $0 . P$ admits maps which are not strong iff there exists a $S$-decomposition $P=U \times V$ of $P$ and elements $u, x, y$ greater than 0 with $u, x \in U, u \diamond x, y \in V$, such that $(x, y) \in P$, while $(u, y) \notin P$.

Proof. Let $P=U \times V$ and elements $u, x, y$ satisfy the hypothesis. Let $\psi=\phi(U, V)$ be the strong map defined by (9). Since $u \diamond x$, (9) implies $\psi(u) \diamond \psi(x, y)$. In $\psi(P)$ we add the relation $\psi(u)<\psi(x, y)$ and all relations arising transitively therefrom. We proceed to verify that the function $\phi: \phi(x)=\psi(x)$ for all $x \in P$ from $P$ onto the partial order $\phi(P) \supset \psi(P)$, thus constructed, is in fact a map. The only way $\phi$ can fail to be segment-preserving is if there exists a segment $R=[(k, l),(m, n)] \subseteq P$ such that $\psi(u), \psi(x, y) \in \psi(R)=[\psi(m, l), \psi(k, n)]$. But in this case (9) implies $y \leqq n, u \leqq m$. Hence since $(m, n) \in P$, we have $(m, y) \in P$ and thus $(u, y) \in P$, a contradiction. Since, by construction of $\phi$, the pre-image of the segment $[\phi(u), \phi(x, y)]$ is just $\{u,(x, y)\}$, the map $\phi$ is not strong.

Conversely suppose the hypothesis fails and let $\phi$ be any map with $[\phi]=[U, V]$. We wish to verify (12). Suppose $(x, y)^{\prime} \leqq(r, s)^{\prime}$ in $\phi(P)$, then by (6)

$$
x^{\prime} \leqq(x, y)^{\prime} \leqq(r, s)^{\prime} \leqq s^{\prime} .
$$

Case a. $(x, s) \in P$. By (6), $\phi[0,(x, s)]=\left[x^{\prime}, s^{\prime}\right]$ hence by (13) $(x, y) \leqq(x, s)$, $(r, s) \leqq(x, s)$, or equivalently $y \leqq s, r \leqq x$. Thus $(r, y) \in P$, and setting $(r, y)=(a, b)$ condition (12) is satisfied.

Case b. $(x, s) \notin P$. First we note $x \neq r, s+y$ and $s>0$. Next if $x \diamond r$, then $x>0$, $r>0$, trivially. But now by hypothesis $(x, s) \in P$, a contradiction. Similarly $y \diamond s$ is impossible. Hence the final possibility is $r<x$ and $y<s$. In this case $(r, y) \in P$ and with $(r, y)=(a, b)$ condition (12) is satisfied.

Figure 1 depicts an example of a partial order with zero-element which admits other than strong maps. By Theorem 3 it is the smallest such example. 

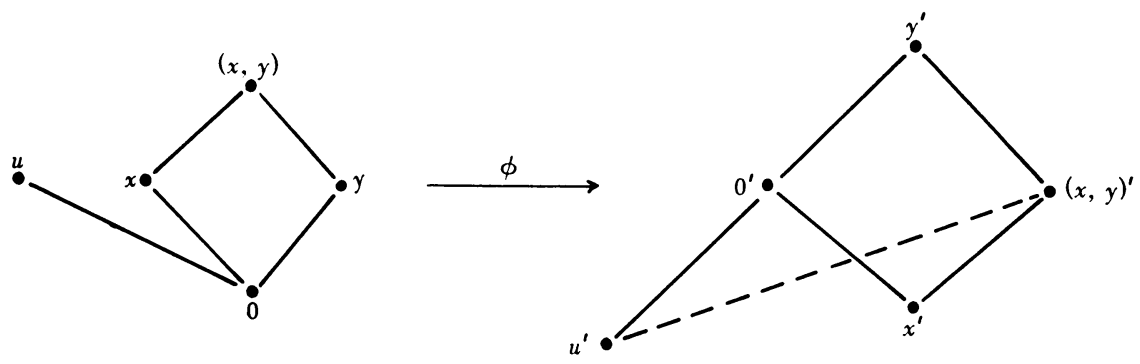

Figure 1

Let us interpret Theorem 3 in terms of the following graph-theoretical concept. With an arbitrary partial order $P$ we associate its incomparability-graph $I(P)$ whose vertex-set $E(P)$ is such that $x$ is adjacent to $y$ in $I(P)$ iff $x \diamond y$ in $P$. If $M, N$ are two components of $I(P)$ and $x \in M, y \in N$ then $x$ and $y$ are comparable and it readily follows from the definition that either $x<y$ for all $x \in M$ and all $y \in N$ or $y<x$ for all such pairs. Hence we may unambiguously define $M<N$ or $N<M$ according to whether $x<y$ or $y<x$ for $x \in M, y \in N$. This way we arrange the components of $I(P)$ into a linear order $\mathscr{C}(P)=\left(C_{i}^{P}: i \in \mathscr{I}\right)$ such that $C_{j}^{P}<C_{k}^{P}$ iff $j<k$. If $P$ has a zero-element 0 (unit-element 1 ), then $\{0\}(\{1\})$ constitutes a component of $I(P)$ and hence is the first (last) member of $\mathscr{C}(P)$.

With these preparations we immediately deduce

Corollary 4. Let $P$ have a zero-element. Then every map $\phi$ on $P$ is strong iff for every S-decomposition $P=U \times V$ of $P$ and every pair $C_{j}^{U} \in \mathscr{C}(U), C_{k}^{V} \in \mathscr{C}(V)$, we either have $(x, y) \in P$ for all $x \in C_{j}^{U}$ and all $y \in C_{k}^{v}$ or $(x, y) \in P$ for no such pair.

Let $P$ have a zero-element 0 and let us denote the unit-element (if it exists) of an arbitrary suborder $K \subseteq P$ by $1_{K}$, with $1_{P}=1$. Assume $P$ only admits strong maps. Let $P=U \times V$ be an arbitrary but fixed $S$-decomposition. If $M \in \mathscr{C}(U), N \in \mathscr{C}(V)$ are such that $(x, y) \in P$ for all $x \in M, y \in N$, we write $M \sim N$, otherwise $M \sim N$. Clearly

(i) $M \sim\{0\}$ for all $M \in \mathscr{C}(U)$,

(ii) $\{0\} \sim N$ for all $N \in \mathscr{C}(V)$,

(iii) $M \sim N$ and $R<M \Rightarrow R \sim N$,

(iv) $M \sim N$ and $S<N \Rightarrow M \sim S$.

If for no choices $M \neq\{0\} \in \mathscr{C}(U), N \neq\{0\} \in \mathscr{C}(V)$ we have $M \sim N$, then $P=U \oplus V$, the direct sum of $U$ and $V$.

Suppose $P \neq U \oplus V$ and assume $P$ can be $S$-decomposed into at least three factors. Then in view of Proposition 6 at least one of $U, V$, say w.l.o.g. $U$, can be $S$-decomposed into two factors $U=R \times S . E(R-\{0\}) \cup E(S-\{0\})$ is contained in a member of $\mathscr{C}(U)$. If some $(r, s) \neq 0 \in U$ is comparable with all of $R$ and $S$, then 
$(r, s) \geqq x$ for all $x \in R$ and for all $x \in S$. Hence $(r, s)=1_{U}, r=1_{R}, s=1_{s}$. Thus $\mathscr{C}(U)$ consists of $\{0\}, U^{\prime}=U-\left\{0,1_{U}\right\}$ and possibly $\left\{1_{U}\right\}$, if $1_{U}$ exists. By assumption and (14) (iii) there exists $N \neq\{0\} \in \mathscr{C}(V)$ with $U^{\prime} \sim N$. Writing $P=R \times W$, where $W=S \times V$ (by Proposition 7, this is possible) we conclude by the same argument that $\mathscr{C}(W)$ consists of $\{0\}, W^{\prime}=W-\left\{0,1_{w}\right\}$ and possibly $\left\{1_{w}\right\}$. Since $N \subseteq V \subseteq W^{\prime}$ $\cup\{0\}$ and $R \subseteq U^{\prime} \cup\{0\}$ we infer in succession:

$$
\begin{aligned}
(x, y) \in P \text { for all } x \in R, y \in N & \Rightarrow(x, y) \in P=R \times W \text { for all } x \in R, y \in W^{\prime} \\
& \Rightarrow(x, y) \in P \text { for all } x \in R, y \in V \\
& \Rightarrow(x, y) \in P \text { for all } x \in U^{\prime}, y \in V .
\end{aligned}
$$

If $1_{U}$ exists we deduce $\left(1_{R}, w\right) \in P=R \times W$ for all $w \in W^{\prime}$ and thus, by Proposition 7, $\left(1_{U}, y\right) \in P=U \times V$ for all $y \neq 1_{V} \in V$. But this means $P=U \otimes V-$ (possibly) $\{1\}$, if 1 exists.

We summarize the results we obtained in the following statement.

THEOREM 4. Let $P$ have a zero-element. Then

(i) $P$ admits only the trivial maps iff it is $S$-indecomposable,

(ii) if for every $S$-decomposition into two factors either $P=U \oplus V$ or $P=U \otimes V$ or $P=U \otimes V-\{1\}$, if 1 exists, then $P$ only admits strong maps,

(iii) if $P$ can be $S$-decomposed into at least three factors, then the converse of (ii) also holds.

By applying Corollary 3 to the preceding theorem and Theorem 2 we obtain

Corollary 5. Let $P$ have a zero-element 0 . If a S-decomposition $P=\prod_{\alpha \in A} P_{\alpha}$ of $P$ into $S$-indecomposable factors $P_{\alpha} \neq\{0\}(\alpha \in A)$ exists, then

(i) $P$ admits only the trivial maps iff $|A|=1$,

(ii) if $P=\prod_{\alpha \in A}^{\oplus} P_{\alpha}$ or $P=\prod_{\alpha \in A}^{\otimes} P_{\alpha}-($ possibly $)\left\{\left(\cdots 1_{p_{\alpha}} \cdots\right)\right\}$, if $\left(\cdots 1_{p_{\alpha}} \cdots\right)$ exists, then $P$ only admits strong maps,

(iii) if $|A| \geqq 3$, then the converse of (ii) also holds,

(iv) there are exactly $2^{|A|}$ strong maps on $P$.

We note that if $P$ contains a unit-element i.e., $P$ is itself a segment, then any $S$-decomposition of $P$ is a direct decomposition, and thus every map on $P$ is strong.

THEOREM 5. Let $P$ have a zero-element 0 . Then

(i) $\phi^{-1}$ is segment-preserving on $\phi(P)$ for any map $\phi$ iff $P=U \otimes V$ for any $S$-decomposition of $P$ into two factors,

(ii) $\phi(P)=Q_{1} \times Q_{2}$ with $Q_{1} \cong \phi(U), Q_{2} \cong \phi(V)$ for any map $\phi$ with $[\phi]=[U, V]$ iff $P$ is a segment.

Proof. To prove (i) let $P=U \times V$ be any $S$-decomposition of $P$ into two factors $U, V$. Let $\phi$ be a map with $[\phi]=[U, V]$ and assume $\phi^{-1}$ is a map on $\phi(P)$. Since then the pre-image of any segment $Q \subseteq \phi(P)$ is a segment in $P$, it, in particular, contains a zero-element $0_{Q}$, and thus $\phi$ is strong. Let $x \in U, y \in V$ be arbitrary and 
suppose $\phi^{-1}\left[x^{\prime}, y^{\prime}\right]=[(k, l),(m, n)]$. Since by $(9) \phi[(k, l),(m, n)]=\left[(m, l)^{\prime},(k, n)^{\prime}\right]$, we infer $x=(m, l), y=(k, n)$. But this means $l=k=0,(m, n)=(x, y) \in P$, and thus $P=U \otimes V$.

Conversely if $P=U \otimes V$ for any $S$-decomposition into two factors, then Theorem 4 implies that every map is strong. Hence if $\phi$ is a map with $[\phi]=[U, V]$, then by (9) $\phi^{-1}\left[(x, y)^{\prime},(r, s)^{\prime}\right]=[(r, y),(x, s)]$, for any segment $\left[(x, y)^{\prime},(r, s)^{\prime}\right] \subseteq \phi(P)$, and $\phi^{-1}$ is a map.

As for (ii) let $\phi$ be the trivial map with $\phi(P) \cong P^{*}$. Since $S$-products are only defined for partial orders with zero-elements, $P^{*} \cong Q_{1}$ must possess a zero-element, and hence $P$ a unit-element.

Conversely, let $P$ be a segment and $\phi$ any map with $[\phi]=[U, V]$. As we remarked above, $\phi$ is strong and hence defined by (9). Setting $Q_{1}=\phi(U), Q_{2}=\phi\left\{\left(1_{U}, y\right): y \in V\right\}$ and defining $\sigma$ on $\phi(P)$ by $\sigma(x, y)^{\prime}=\left(x^{\prime},\left(1_{U}, y\right)^{\prime}\right)$, it readily follows from (9) that $\sigma$ is the desired isomorphism from $\phi(P)$ onto $Q_{1} \times^{\phi(P)} Q_{2}$.

We lastly apply Corollary 3 to the preceding theorem, and obtain

Corollary 6. Let $P$ have a zero-element 0 . If a $S$-decomposition $P=\prod_{\alpha \in A} P_{\alpha}$ of $P$ into $S$-indecomposable factors $P_{\alpha} \neq\{0\}(\alpha \in A)$ exists, then

(i) $\phi^{-1}$ is segment-preserving on $\phi(P)$ for any map $\phi$ iff $P=\prod_{\alpha \in A}^{\otimes} P_{\alpha}$,

(ii) $\phi(P)=\prod_{\alpha \in A} Q_{\alpha}$ with $Q_{\alpha} \cong \phi\left(P_{\alpha}\right)$ for any map $\phi$ is the $S$-decomposition of $\phi(P)$ into $S$-indecomposable factors $\neq\{0\}$ iff $P$ is a segment.

Acknowledgments. We would like to express our thanks to G.-C. Rota and R. Stanley for calling this problem to our attention and to A. M. Gleason for a stimulating discussion.

\section{REFERENCES}

1. G. Birkhoff, Lattice theory, Amer. Math. Soc. Colloq. Publ., vol. 25, Amer. Math. Soc., Providence, R. I., 1940; 2nd rev. ed., 1948; 3rd ed., 1967. MR 1, 325; MR 10, 673; MR 37 \#2638.

Mathematisches Institut, Universität Tübingen, 74 Tübingen, Federal Republic of GermanY

Department of Mathematics, Wayne State University, Detroit, Michigan 48202 